\title{
Individual management of cervical cancer during pregnancy
}

\author{
Yan Qin Zhang ${ }^{1}$, Di Wu ${ }^{1}$, Meng Qi Deng ${ }^{1}$, Xiang Yu Chang ${ }^{1}$, Jin Wei Miao ${ }^{1}$, Yu Mei Wu ${ }^{1}$ \\ ${ }^{1}$ Department of Gynecologic Oncology, Beijing Obstetrics and Gynecology Hospital, Capital Medical University, Beijing, 100006, P. R. China
}

\begin{abstract}
Summary
The exact definition of cervical cancer during pregnancy has not yet been unified. Cervical cancer during pregnancy refering to cervical cancer found during pregnancy is widely accepted. With the phenomenon of late marriage and late childbearing globally, the incidence of cervical cancer during pregnancy is also increasing. Even though early screening for cervical cancer has had an enormous impact on incidence and mo rtality from cervical cancer worldwide, cervical cancer during pregnancy is still a major clinical problem due to the negligence and misunderstanding of early symptoms. At present, there is no global guideline for the treatment of cervical cancer during pregnancy. The clinicians can only treat according to there own experience and the patient's wishes. Here, we provide five cases of cervical cancer during pregnancy in our hospital.
\end{abstract}

Key words: Cervical cancer during pregnancy; Treatment; Chemotherapy; Case.

\section{Introduction}

Cervical cancer (CC) is one of the most frequent types of cancer in women worldwide [1]. Accordingly, $\mathrm{CC}$ is one of the most three common pregnancy-associated cancer types $[2,3]$, and about $3 \%$ of the cases are diagnosed during pregnancy $[4,5]$. Although cervical cancer during pregnancy is common and some data suggest that diagnosis during pregnancy does not affect survival rates negatively [6], its treatment is still a problem. As cervical cancer during pregnancy presents patients and physicians with a dilemma because the negative treatment can seriously threaten the health of the mothers, while positive treatment may carry the risk of losing the fetuses. The management for this situation mainly depends on the gestational age at the time of diagnosis, disease staging, size of the lesion and the patient's wish to maintain pregnancy and fertility [7]. To provide a reference for others, we present five reference cases diagnosed and treated with CC during pregnancy. In all patients, colposcopy-directed biopsies of suspicious lesions were undertaken. Tumor grade was determined based on the modified Broders' classification [8], tumors were staged clinically according to the International Federation of Gynecology and Obstetrics (FIGO) system [9], the lymph node status was recorded separately.

\section{Cases Presentation}

The first patient is a 36yearold woman, gravida 1 , para 0 , with a 2year history of infertility underwent a course of IVF (January 30, 2018) with two fresh embryos and one to survive. In early pregnancy, there was a small amount of blood discharge, and progesterone was given orally to $3+$ months. Vaginal bleeding occurred again during $4+$ months of pregnancy. Ultrasound examination of the placenta showed no abnormalities, but colposcopy with cervical biopsy showed a cervical squamous cell carcinoma. High-risk human papillomavirus (HPV) was positive and pelvic Magnetic Resonance Imaging (MRI) result indicated that cervical tumor invaded to cervical muscle layer, the left fornix and vaginal wall were partially eroded and cervical squamous cell carcinoma was detected on pathological biopsy. Therefore, the final diagnosis is cervical squamous cell carcinoma stage IIA2 G2. After been fully informed of the disease, the patient and her family requested that the fetus be retained. On June 21, 2018 and July 27, 2018, neoadjuvant chemotherapy with PT regimen was given once respectively. Specific regimens: paclitaxel liposome $240 \mathrm{mg}$, cisplatin $120 \mathrm{mg}$. During that period, the patient underwent a normal maternity check-up. Dexamethasone sodium acetate was used to enhance maturation of the fetal lung at 32 weeks of gestation. The patient underwent cesarean section and radical resection of cervical cancer at 34 weeks plus 3 days of gestation in our hospital. She successfully gave birth to a boy, weight $2600 \mathrm{~g}$. The Apgar score was 10 points at 1,5 and 10 minutes. Postoperative pathological examination revealed a cervical squamous cell carcinoma, nonkeratinized, with a depth of invasion greater than $2 / 3$ of the wall thickness of the cervical canal, vaginal wall involvement, three metastases found in 22 lymph nodes (Figure 1-A). After surgery, PT regimen was first given (270 $\mathrm{mg}$ of paclitaxel and $120 \mathrm{mg}$ of nedaplatin) on October 9 , 2018, and the second (300 $\mathrm{mg}$ of paclitaxel and $120 \mathrm{mg}$ of nedaplatin) on November 2, 2018. At present, with regular outpatient review, the general condition of the patient and the growth of newborn is going well without any obvious abnormal performance.

The second patient is a 38 yearold woman, gravida 2, para 0 , due to secondary infertility she underwent a course of IVF (September 4 2017) with 2 fresh embryos and one survived. Pregnancy-saving treatment with progesterone
Eur. J. Gynaecol. Oncol. - ISSN: 0392-2936 XLI, n. 5, 2020

doi: $10.31083 /$ j.ejgo.2020.05.5214
This is an open access article under the CC BY 4.0 license (https://creativecommons.org/licenses/by/4.0/). 
Table 1. - Basic information of five cases

\begin{tabular}{lccccccccccc}
\hline Cases & Age & HPV & Sym & GA & Sta & His & TDP & Tre & Pro & Lym-Met & Follow up (Mths) \\
\hline $\mathbf{1}$ & 36 & HR & Vb & 28 & IIA2 G2 & SCC & Yes & Nc+Sur+Ch & Bh+NED & Have & 12 \\
$\mathbf{2}$ & 38 & 16 & Vb & 14 & IIB2 G2 & SCC & Yes & Nc+Sur+Ch & Bh+NED & Have & 19 \\
$\mathbf{3}$ & 34 & HR & Cb & 16 & IB1 G2 & SCC & Yes & Sur+Ch & NED & No & 17 \\
$\mathbf{4}$ & 38 & ND & Vb & postnatal & IIA2 G2 & SCC & No & Sur+Ch & Relapse & Have & 25 \\
$\mathbf{5}$ & 31 & ND & Vb & postnatal & IIBG2 G2 & ACC & No & Ch & Death & Have & 5 \\
\hline
\end{tabular}

HPV: human papillomavirus, Sym:symptoms, GA: gestational age, Sta: Stage, His: Histology, TDP: therapy during pregnancy, Tre: treatment, Pro: Prognosis, Lym-Met: Lymph node metastasis, HR: high risk, Vb: Vaginal bleeding, Cb: Contact bleeding, $\mathrm{Nc}+$ Sur $+\mathrm{Ch}$ : Neoadjuvant chemotherapy + surgery + chemotherapy, Bh: Baby health, NED: no evidence of disease, ND: not determined, SCC: squamous cell carcinoma, ACC: adenosquamous cell carcinoma, Mths: months.

was given in the early stages of pregnancy. Vaginal intermittent bleeding occurred at $8+$ weeks of gestation, and cervical neoplasms were found at $12+$ weeks of gestation. HPV was tested positive for type 16 and heterosexual cell clusters could be detected by liquid-based thin-layer cytology (TCT). The consultation of pathology indicated that it is cervical squamous cell carcinoma of non-keratinized type with poor differentiation. MRI findings revealed an exogenous mass of cervical anterior labial margin, malignant tumor was considered, which invaded probably to grassroot level, multiple pelvic large lymph nodes were found, metastasis could not be excluded. Therefore, the final diagnosis was cervical squamous cell carcinoma stage IIB2 G2. After fully been informed of the disease, the patient and her family requested that the fetus be retained. On December 29, 2017 and January 22, 2018, PT regimen neoadjuvant chemotherapy was given once respectively. Specific regimens: paclitaxel liposome $210 \mathrm{mg}$, cisplatin 100 $\mathrm{mg}$. After promoting maturation of the fetal lung, cesarean section and bilateral ovarian biopsy were performed at 33 weeks of gestation. Finally she gave birth to a girl weighing $2100 \mathrm{~g}$, the Apgar score was all 10 points at 1 minute, 5 minutes, 10 minutes. Postoperative ovarian pathology suggested that there was no metastasis in bilateral ovaries. She was treated in our hospital for radical operation of cervical cancer and bilateral ovarian suspension 2 months after operation. Pathological reports of postoperative was obtained as cervical squamous cell carcinoma, non-keratinized, with a depth of invasion greater than $1 / 3$ and less than $2 / 3$ cervical canal wall thickness (Figure 1-B). Two metastatic lymph nodes were found in the right lateral iliac lymph nodes. The patient is still recieving radiotherapy in our hospital, the general condition of the patient and the growth of newborn are going well.

The third patient is a 34yearold woman, gravida 3, para 1. The patient underwent an induced abortion in 2006 and 2008 respectively. The date of last menstrual period was on November 9, 2017. Gynecological examination showed a large erosion of the cervix with severe bleeding after contacting, and a swelling and hardening posterior lip. TCT test revealed a high degree of squamous intraepithelial lesions. Colposcopic pathological results showed CIN III on 1, 2, 5,11 points, and 2,5 points were partially invasive squa- mous cell carcinomas. MRI findings revealed an abnormal singnals on anterior and inferior lip of cervix, cervical cancer was suspected. Therefore, the final diagnosis was cervical squamous cell carcinoma stage IB1 G2. After been fully informed of the disease, the patient and her family requested active treatments of the disease instead of retaining the fetus. Under the condition of abdominal aortic balloon implantation, radical operation for cervical cancer plus bilateral ovarian biopsy and suspension were performed on April 2, 2018. Postoperative pathology showed invasive squamous cell carcinoma of the cervix, non-keratinized type, less than $1 / 3$ of the wall thickness of cervical canal, intravascular cancer tissue, no lymph node metastasis (Figure 1-C). On April 232018 and May 25 2018, PT regimen chemotherapy was given once respectively. Specific regimens: paclitaxel liposome $240 \mathrm{mg}$, cisplatin $110 \mathrm{mg}$. Now the patient is in good condition.

The fourth patient is a 38yearold woman, gravida 4, para 1. Cervical ectopic neoplasms were found after cesarean section on reexamination. On retrospective medical history analysis of the patient, intermittent vaginal bleeding and postcoital bleeding were found during pregnancy. However, these symptoms were not taken seriously at that time, and were treated as threatened abortion and pregnancysaving treatment was given. To make matters worse, TCT and gynecological tests did not perform throughout pregnancy. Colposcopic pathology revealed a possibility of 3,6 , 9 and 12 cervical moderately differentiated mucinous squamous cell carcinoma. The MRI found a solid tumor in the lower cervix and multiple swollen lymph nodes in the pelvic cavity, then cervical cancer with pelvic metastasis was considered. Therefore, the final diagnosis is cervical squamous cell carcinoma stage IIA2 G2. After been fully informed of the disease and surgical risk radical operation for cervical cancer was done on June 13, 2018. Postoperative pathological demonstrated an indifferentiated carcinoma in uterus which was larger than $1 / 3$ and less than $1 / 2$ wall thickness. The differentiation of squamous cell carcinoma was partially considered, and lymph node metastasis in right iliac fossa could be seen ( Figure 1-D). Postoperatively, PT regimen chemotherapy was given once respectively on July 7, 2018 and August 9, 2018. Specific regimens: paclitaxel liposome $240 \mathrm{mg}$, cisplatin $110 \mathrm{mg}$ and Nedaplatin- 

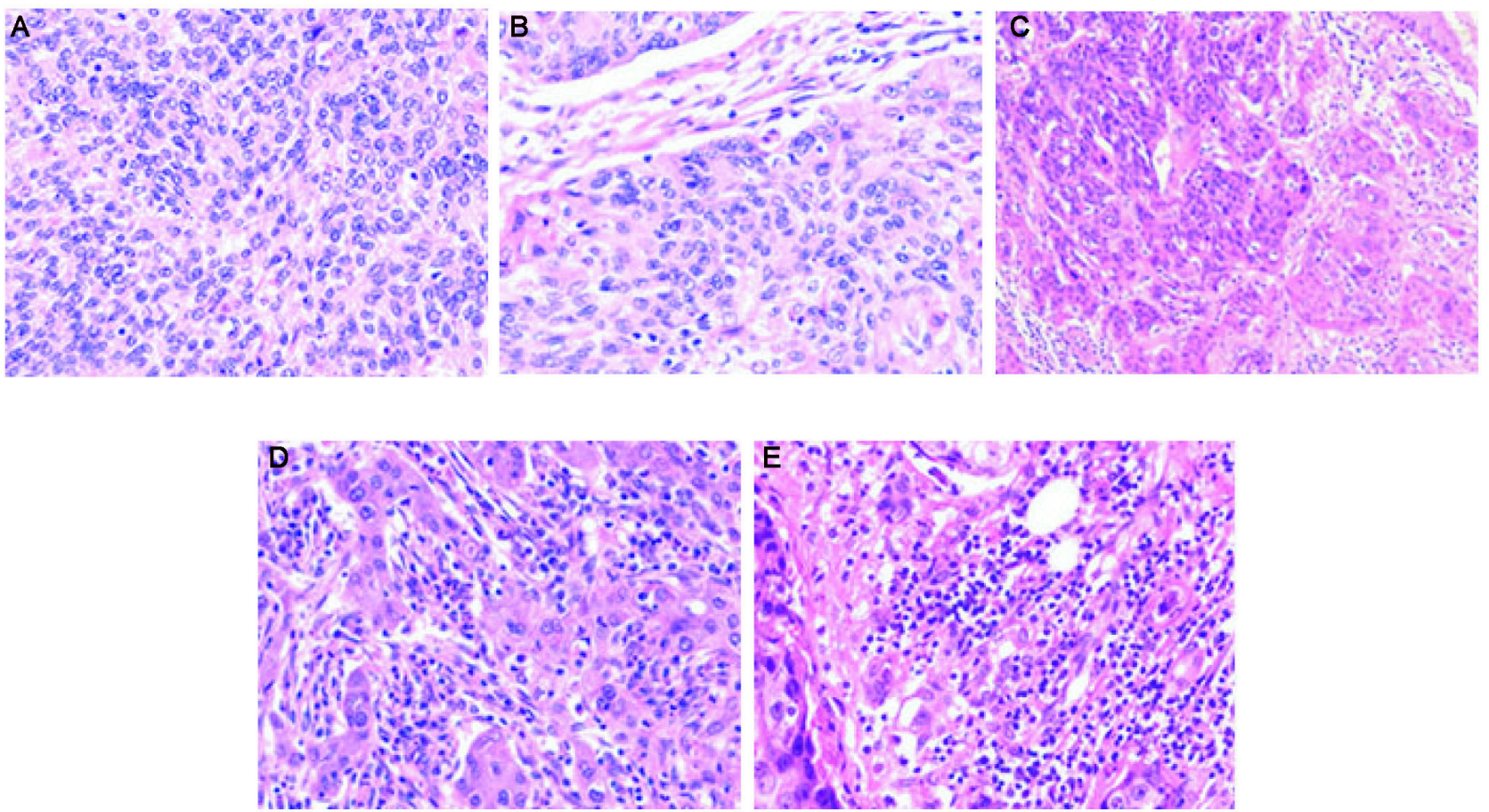

Figure 1. - Postoperative pathological charts of 5 cases of cervical cancer during pregnancy. The cells with enlarged nuclei are cancer cells, and the nuclei are stained with obvious blue. A: Pathology of first case, B: Pathology of second case, C: Pathology of third case, D: Pathology of fourth case and E: Pathology of lymph node metastasis of fifth case. Scale bar $=50 \mu \mathrm{m}$.

sensitized chemotherapy was supplemented three times in consideration of lymph node metastasis. At present, the patient's general physical condition is good with routine outpatient reexamination.

The fifth patient is a 31 yearold woman, gravida 1, para 1. Cervical ectopic neoplasms were found after cesarean section on reexamination. On retrospective medical history analysis of the patient, vaginal bleeding and increased secretions were appeared in third months of pregnancy. She was treated with fetal protection in the local hospital. The vaginal secretion further increased in 8 months of gestation, and cervical tumors were found in local hospital. However, no treatment was given, but postpartum reexamine was recommended. Cervical adenosquamous carcinoma was revealed by colposcopic biopsy at 42 days after delivery. Detailed gynecologic examination revealed an exogenous cervical mass, $6 \mathrm{~cm}$ in diameter, brittle and bleeding, normal sized uterus, right parauterine elastic nodule thickening to the pelvic wall. The final diagnosis is adenosquamous carcinoma of cervix stage IIIB G2. According to NCCN guidelines, stage III cervical cancer has lost the chance of radical treatment. After been fully informed of the disease and advantages or disadvantages of all treatment schemes. The patient finally underwent bilateral ovarian suspension surgery and pelvic lymph node dissection in our hospital. Postoperative pathological examination reported a widespread metastasis was seen in lymph nodes (Figure 1-E). Then she was given adjuvant radiotherapy and chemotherapy after operation. However, one month after the operation, the patient faced tumor spread, with type 2 diabetes mellitus leading poor prognosis. Later, only symptomatic supportive treatment was given, and the patient eventually died of cervical cancer.

Analysis of the five cases, median age at diagnosis was 35 years (rang from 31 to 38 ). All the patients have the symptoms of vaginal bleeding in early stages of pregnancy. Cervical cancer was found by further examination in three patients, and were given active treatment. Two of three who received active treatment chose to retain the fetus and neoadjuvant chemotherapy were given twice PT regimen respectively. They were also given active treatment for cervical cancer after cesarean section. The results of the cases showed that the neonatal Apgar score of two children whose mothers receivied neoadjuvant chemotherapy during pregnancy were all 10 at 1 minute, 5 minutes and 10 minutes. At present, the two newborns are living well and have no obvious effect on the later stage of chemotherapy. For the two pregnant women who chose to keep the fetus, the cesarean section was completed after the fetus lung maturation was promoted at 34 weeks of gestation and 33 weeks of gestation. Two patients with negative treatment were not confirmed by biopsy because their negative treatment attitude and medical misdiagnosis. The discovery of cervical neoplasms and subjective neglect, without further pathological diagnosis directly lead to delays in providing passive treatment and improvement of condition. 


\section{Discussion}

Although the prevention and screening methods for cervical cancer have been increasing in recent years. As more and more women choose to delay the marriage and have children lately, even a further rise of gestational $\mathrm{CC}$ is plausible [2], but the body of literature addressing it is still scarce. Therefore, we summarize the analysis of pregnancy-related cervical cancer cases in clinical work and provide reference materials for their treatment. Although cervical cancer is virtually preventable by HPV and effective screening strategies, its peak occurrence coinciding with vaginal bleeding in pregnant women can be subjectively judged as pregnancy-related bleeding increasing the risk of locally advanced cervical cancer in pregnancy. According to the statistics, $\mathrm{CC}$ is one of the three most common pregnancy-associated cancer types. With the prevalence of late marriage and late childbearing, timely discovery and treatment of cervical cancer in pregnancy of elderly primipara has become an urgent problem that needs to be solved. Through the analysis of 5 cases of cervical cancer during pregnancy in our hospital, we found that vaginal bleeding is a prominent clinical manifestation of pregnancy with cervical cancer. Therefore, we suggest that, a pregnant women with long-term intermittent vaginal bleeding, especially HPV positive, should be examined by TCT. Patients with abnormal cytological test results should be subjected to colposcopy, which can decide whether a biopsy is needed and which site is the best. Colposcopy can also rule out or confrm the presence of microinvasion or invasion, thereby defniing the type of treatment and the delivery timing and route. Some studies believe that prolongation of pregnancy at an early stage and thus delaying definite treatment was safe [10]. Some cervical cancers during pregnancy can regress and disappear after delivery. However, some cervical cancer during pregnancy often progress rapidly, and positive clinical treatment has a great impact on the prognosis of patients, regardless of the patient decisions to continue pregnancy or not [11]. Also, some articals reported that positive treatment and trying to prolong the pregnancy did not have a detrimental effect on the mother's prognosis [12]. Ignorance of the disease during pregnancy or desiring to treat after childbirth can not be recommended. Therefore, we emphasize that for cervical cancer found in the early stages of pregnancy, no matter the patients desire to preserve the fetus or not, they should take active treatments such as adjuvant chemotherapy. Nowadays, the advantages of neoadjuvant chemotherapy in pregnancy complicated with cervical cancer to allow pregnancy and control the development of disease have been widely recognized [13]. Platinum was shown to be a safe option during pregnancy [14], especially since platinum concentrations are extremely low in the fetal unit suggesting placental filtration [15]. Even if there are no standardized practices in the treatment of cervical cancer in pregnancy, neoadjuvant chemotherapy can be a very useful strategy for patients and physicians facing the challenge. For the two cases reported in this article, both patients experienced a dramatic reduction in tumor volume, and there were no adverse fetal effects, and favorable neonatal outcomes were achieved. The choice of delivery time still requires a comprehensive assessment of the condition during pregnancy. In accordance with current guidelines, preterm labor was tolerated in our cohort to compromise between fetal maturity and completion of the mothers' oncological treatment [16]. Undoubtedly, the most successful strategy against CC during pregnancy is the participation in preconceptive cancer screening programs. We strongly recommend that all pregnant women plan pre-pregnancy checkup, especially for pregnant women older than 35 years. Vaginal bleeding during pregnancy must been taken seriously. Colposcopy-directed biopsy is a safe and reliable procedure during pregnancy. Delayed bleeding can occur but is often successfully resolved with the application of pressure [17].

\section{Conclusions}

The author analyzed the clinical cases of these five pregnancy with cervical cancer, and the treatment points are summarized as follows: Vaginal bleeding is a prominent clinical manifestation of cervical cancer during pregnancy. We suggest that pregnant women with long-term intermittent vaginal bleeding, especially HPV positive, should be examined by TCT. Patients with abnormal cytological test results should be subjected to colposcopy. We emphasize that for cervical cancer found in early pregnancy, if pregnant women have the desire to preserve the fetus, they should also take active treatments such as adjuvant chemotherapy. The choice of delivery time still requires a comprehensive assessment of the condition during pregnancy. In accordance with current guidelines, preterm labor was tolerated in our cohort to compromise between fetal maturity and completion of the mothers' oncological treatment.

\section{Ethics approval and consent to participate}

We certify that we have obtained all the appropriate patient consent forms. The patients have given their consent for their images and other clinical information to be reported in the journal.

\section{Acknowledgements}

This work was supported by Beijing Municipal Administration of Hospitals Clinical Medicine Development of Special Funding Support (Grant No: ZYLX201705).

\section{Conflict of Interest}

There are no conflicts of interest.

Submitted: March 31, 2019

Accepted: June 05, 2019

Published: October 15, 2020 


\section{References}

[1] Gustafsson L., Pontén J., Bergstrôm R., Adami H.: “International incidence rates of invasive cervical cancer before cytological screening". Int. J. Cancer, 1997, 71, 159-165.

[2] Eibye S., Kjær S.K., Mellemkjær L.: "Incidence of PregnancyAssociated Cancer in Denmark, 1977-2006". Obstetrics \& Gynecology, 2013, 122, 608-617.

[3] Frédéric, A., Fumagalli, M., Dahl, S.K., Lok, C., Van, C.K., Han, S.D., et al.: "Gynecologic cancers in pregnancy: guidelines of a second international consensus meeting. Department of Oncology, Katholieke Universiteit Leuven and Gynecologic Oncology, University Hospitals Leuven”. Int. J. Gynecol. Cancer., 2014, 24, 394.

[4] Traen K., Svane D., Kryger-Baggesen N., Bertelsen K., Mogensen O.: "Stage Ib cervical cancer during pregnancy: planned delay in treatment-case report”. Eur. J. Gynaecol. Oncol., 2006, 27, 615617.

[5] Demeter A., Sziller I., Csapó Z., Szánthó A., Papp Z.: “Outcome of pregnancies after cold-knife conization of the uterine cervix during pregnancy". Eur. J. Gynaecol. Oncol., 2002, 23, 207-210.

[6] Germann N., Haie-Meder C., Morice P., Lhomme C., Duvillard P., Hacene K., et al.: "Management and clinical outcomes of pregnant patients with invasive cervical cancer". Ann. Oncol., 2005, 16, $397-$ 402.

[7] Van Calsteren K., Vergote I., Amant F.: "Cervical neoplasia during pregnancy: Diagnosis, management and prognosis". Best Practice \& Research Clinical Obstetrics \& Gynaecology, 2005, 19, 611-630.

[8] Broders A.C.: "EPITHELIOMA OF THE GENITO-URINARY ORGANS". Ann. Surg., 1922, 75, 574-604.

[9] Hacker N.F.: "Revised FIGO staging for carcinoma of the vulva" International Journal of Gynecology \& Obstetrics, 2009, 105, 105106.

[10] Germann N., Haie-Meder C., Morice P., Lhomme C., Duvillard P., Hacene K., et al.: "Management and clinical outcomes of pregnant patients with invasive cervical cancer". Ann. Oncol., 2005, 16, $397-$ 402.
[11] Kyrgiou M., Tsoumpou I., Vrekoussis T., Martin-Hirsch P., Arbyn M., Prendiville W., et al.: "The up-to-date evidence on colposcopy practice and treatment of cervical intraepithelial neoplasia: The cochrane colposcopy \& cervical cytopathology collaborative group (C5 group) approach". Cancer Treat. Rev., 2006, 32, 516-523.

[12] Van Calsteren K., Vergote I., Amant F.: "Cervical neoplasia during pregnancy: Diagnosis, management and prognosis". Best Practice \& Research Clinical Obstetrics \& Gynaecology, 2005, 19, 611-630.

[13] Van Calsteren K., Vergote I., Amant F.: "Cervical neoplasia during pregnancy: Diagnosis, management and prognosis". Best Practice \& Research Clinical Obstetrics \& Gynaecology, 2005, 19, 611-630.

[14] Zagouri F., Sergentanis T.N., Chrysikos D., Bartsch R.: "Platinum Derivatives During Pregnancy in Cervical Cancer". Obstetrics \& Gynecology, 2013, 121, 337-343.

[15] Köhler C., Oppelt P., Favero G., Morgenstern B., Runnebaum I., Tsunoda A., et al.: "How much platinum passes the placental barrier? Analysis of platinum applications in 21 patients with cervical cancer during pregnancy". am. J. Obstet. Gynecol., 2015, 213, 206.e1-206.e5

[16] Amant F., Van Calsteren K., Halaska M.J., Beijnen J., Lagae L., Hanssens M., et al.: "Gynecologic Cancers in Pregnancy". Int. J. Gynecol. Cancer, 2009, 19, S1-S12.

[17] Woodrow N., Permezel M., Butter-field L., Rome R., Tan J., Quinn M.: "Abnormal Cervical Cytology in Pregnancy: Experience of 811 Cases". the Australian and New Zealand Journal of Obstetrics and Gynaecology, 1998, 38, 161-165.

Corresponding Author:

JIN WEI MIAO Ph.D.

Department of Gynecologic Oncology, Beijing Obstetrics and Gynecology Hospital, Capital Medical University, Qihelou Str.17, Dongcheng District, Beijing, 100006 (China) Email: miaojinweigyn@163.com 